\title{
Automated Haze Removal and Radiometric Normalization for Electro- Optical imagery Preprocessing
}

\author{
Yun Zhan ${ }^{1,}$, Shengchun Zheng ${ }^{1,}$, , Yong $\mathrm{Du}^{2, \mathrm{~b}}$, Luqing Wei ${ }^{1, \text { a }}$ \\ ${ }^{1}$ Qingdao Geotechnical Investigation\&Surveying Research Institute, Qingdao, P. R. China \\ ${ }^{2}$ KSKY Hi-Tech Corp., Gatineau, QC, Canada \\ aaixin70@163.com, ${ }^{b}$ yong.du@ksky.ca
}

Keywords: Automation, Multiresolution, Topology, Adjustment, Normalization.

\begin{abstract}
Two precedures are presented for image preprocessing, automated haze removal and the relative radiometric normalization of multitemporal optical images, to act as the data support for land cover change detection and image analysis. The developed algorithm for haze removal involves processes of feature and texture analysis of the multiresolution spatial frequency distribution of pixel brightness information content of a scene. The image contaminated by haze is decomposed into layers of different resolutions of spatial distribution frequencies. The radiometric characteristics of the corresponding layers are estimated and analyzed with topology based multiresolution spatial analysis technology. Based on the analysis, the haze component is then separated from the remaining spatial frequency components representing spectral information of actual land cover types in the scene, and a spectrally corrected image with "haze-off" characteristics is obtained. Then a method is used for radiometric normalization between multitemporal images of the same area. Case study using several different type images of Qingdao City in China proves the effectiveness of this technique except for those regions too hazy.
\end{abstract}

\section{Introduction}

Highly detailed Electro optical (EO) image data are the primary data source for accurate remote sensing applications in different fields. Undoubtedly, EO image data are usually degraded by the turbid medium (e.g., particles, water-droplets) in the atmosphere, thus availability of a high-quality image often depends on luck. Cloud and haze are two atmospheric effects that cause image contamination. Cloud cover blocks almost all reflected radiation from the surface so substitution using another image is the only information loss recovery method available[1]. Haze partially obscures the ground, so it is theoretically possible to be removed using atmospheric correction techniques.

Haze removal (or dehazing) is highly desired in remote sensing applications, and image processing and analysis. On one hand, it will help making huge amounts of hazy EO image data accessible again as valuable sources of Earth observation information, instead simply being rendered useless and discarded. On the other hand, a satisfactory solution for haze detection and removal will significantly improve the quality of visual image analysis, and even more the accuracy of automated information extraction from EO imagery.

Therefore, many researchers put considerable effort into this topic developing different methods. Generally speaking, there are two haze removal strategies: absolute and relative atmospheric calibration. These approaches have different advantages and shortcomings. A major disadvantage of atmospheric absolute calibration is that they require coincident auxiliary information $[2,3,4]$ based on the measurements taken from different sensors, or individual spectral channels, so the atmospheric effects could be calibrated completely. While the use of in situ information might be the most accurate in terms of correcting for atmospheric haze effects, most users must work with remotely sensed data that have already been collected and therefore, do not have access to such information. The relative calibration is focused on the radiometric relation between multitemporal or multichannel images, or the spatial frequency distribution relation of the regional characteristics, to detect and estimate haze for haze removal in the EO image, such as Dark-target 
deduction[5,6], haze optimized transformation with dark object subtraction[7,8],Histogrammatching approaches[9,10] and corrections using a multitemporal reference image[11,12,13]. Although some relative calibration approaches for haze detection and removal have been developed, they are only available for some special cases, and are not suited for automated processing operations. Truly suitable approaches for fully automated haze detection and removal are not available to date. On the other hand,the value of satellite data in Earth observation for environmental monitoring is well established. With temporal repeat observation, radiometric consistency is hard to maintain between separate images due to different atmospheric conditions, variations in the solar illumination angles, and sensor calibration trends.

Therefore, in the present study, employing topology based multiresolution (or multi-scale) spatial analysis technology and relative radiometric normalization technology[14], the image preprocessing system is developed including two sections: Haze removal and radiometric normalization. Both visual inspection and statistical accuracy assessment show that the algorithms and software are valid and robust.

\section{Technology and method}

Multi-scale spatial analysis technology.Multi-scale spatial analysis technology provides an approach to decompose the image into different levels of spatial scale, meaning scales, or levels, of the distribution pixel value occurrence frequencies. Thus, the features and textures in the image can be separated into individual scale layers of information encrypted in spatial and radiometric pixel frequency distribution for further analysis.

For 2D case, with multi-scale spatial analysis technology, decomposition of a digital image $\left\{C_{n, m}^{0}\right\}_{n, m \in z}$ can be formulated. If the total number of decomposition levels is $N$, the formulate of decomposition of level $k$ are:

$$
\begin{gathered}
C_{n, m}^{k}=\sum_{j, l \in Z} \bar{h}_{j-2 n} \bar{h}_{l-2 m} C_{j, l}^{k-1} \quad d_{n, m}^{k 1}=\sum_{j, l \in Z} \bar{h}_{j-2 n} \bar{g}_{l-2 m} C_{j, l}^{k-1} \quad d_{n, m}^{k 2}=\sum_{j, l \in Z} \bar{g}_{j-2 n} \bar{h}_{l-2 m} C_{j, l}^{k-1} \\
d_{n, m}^{k 3}=\sum_{j, l \in Z} \bar{g} \bar{g}_{j-2 n} C_{l-2 m}^{k-1} \\
k=1,2, \ldots, N .
\end{gathered}
$$

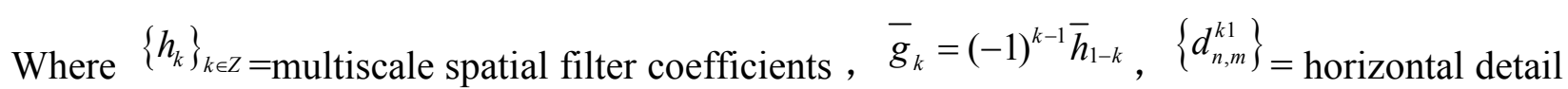
of $k_{\text {level, }}\left\{d_{n, m}^{k 2}\right\}=$ vetical detail, $\left\{d_{n, m}^{k 3}\right\}=$ diagonal detail, $\left\{C_{n, m}^{k}\right\}=$ low frequencies.

Generally, haze has lower frequencies of spatial scale, which would result in relative brighter patterns in haze areas due to the scattering effects. When the lower frequencies of the spatial scale are analyzed in the image, both haze's and ground target's lower frequencies distributions, and the subtle differences or different components between them, should be considered. In order to detect and remove the haze from the contaminated image, the two components of lower frequencies should be separated. A haze free image of the same area could be the reference to subtract the lower frequencies distributions of the ground[11,12]. But a haze removal algorithm which requires no reference image has been the goal for this study, which will be more convenient and could be run as a fully automated process, especially with no haze free reference image available.

Topology method. Topology is the combination of geometry and a theory needed to study spatial relations within the image. Traditionally the whole spatial properties of an image are collected and analyzed. With topological analysis, the specific structures in an image can be selected and analyzed locally. The statistical characteristics and other properties of such local areas, therefore, can be focused on, and analyzed in more detailed. To analyze the spatial characteristics within an image by means of algebraic operations, quantifying the image should be the first step. In the present study, quantifying the topological characteristics (features, textures and other regional 
properties, etc.) of images, not just the individual pixel, is the objective. The multi-scale spatial analysis technology decomposes the image into different spatial scale layers, and separates the different scales of the spatial characteristics. Therefore, the homology groups can be computed and analyzed through topological analysis. The structures record the way the image is partitioned into components and capture tunnels, voids, and other higher dimensional features of the image. This decomposition by using topology-based multiscale spatial analysis technology provides a starting point for image enhancement and simplification for further feature extraction and analysis. Combined with multi-scale spatial analysis technology, the topology of the space of $n$ by $n$ pixel patches is improved to adapted-areas of the local regions.

In the present study, the lower frequencies of spatial scale components in the image are emphatically concerned for haze layers detection and extraction. After the decomposition, the brightness of the lower frequencies is analyzed to separate the haze components from the other lower frequency distribution of actual ground surface features. The algorithms developed in the present study are proven efficient and robust.

In practice,the Automated Adaptive Haze Removal System has two workflows: full automation for basic users, and adjustment for advanced users(Fig. 1).

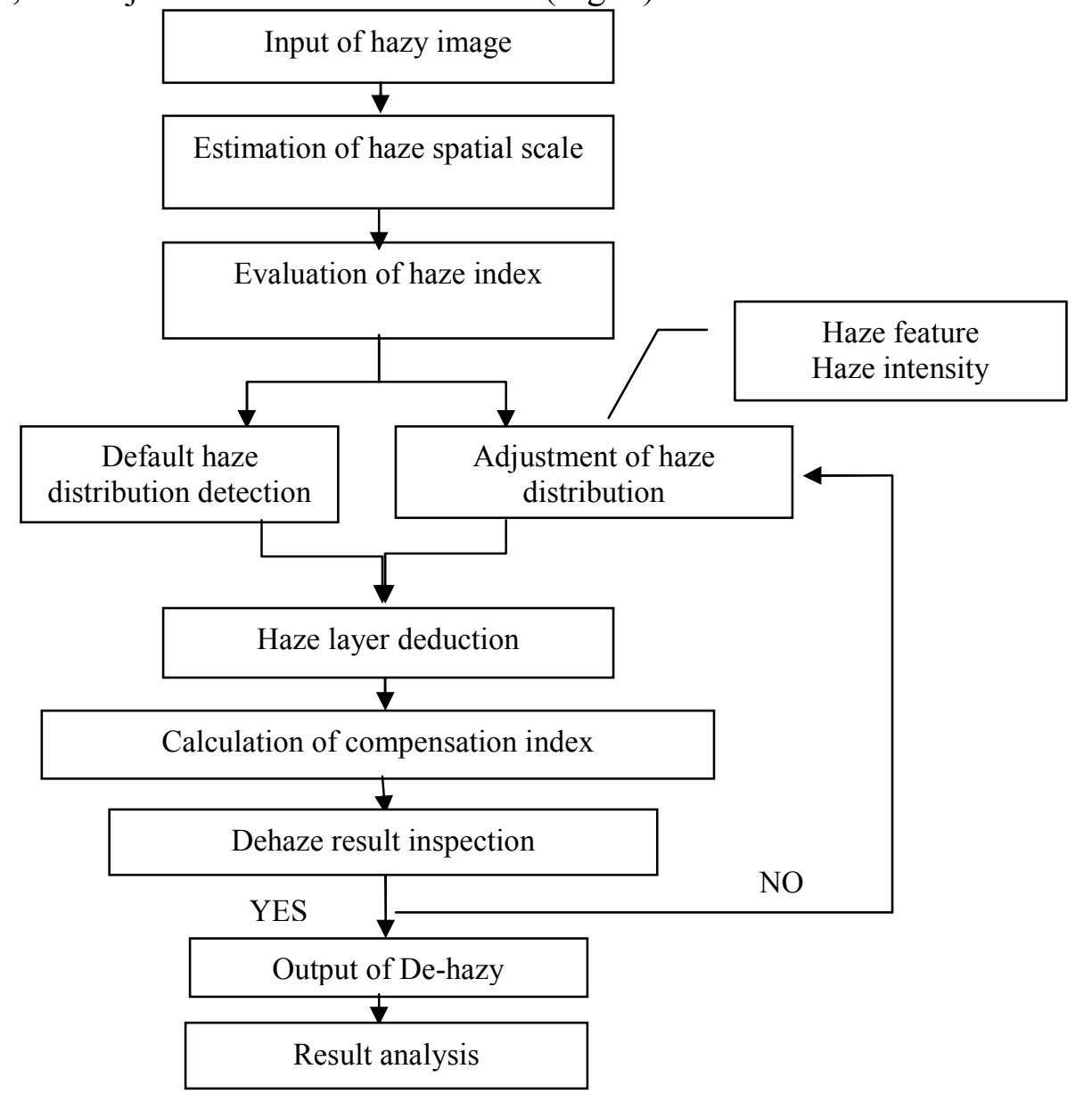

Fig.1. Flowchart of haze removal

Relative radiometric normalization technology. The radiometric normalization of multitemporal electro-optical images of the same terrain is often necessary for land cover change detection. This article uses the procedure for radiometric normalization between multitemporal images of the same area[14]. The selection of pseudo-invariant features(PIFs) is done statistically. With quality control, principal component analysis (PCA) is used to find linear relationships between multitemporal images of the same area. The images are normalized radiometrically to a common scale tied to the reference radiometric levels.The procedure(Fig.2) ensures the conservation of radiometric resolution for the multitemporal images involved. 


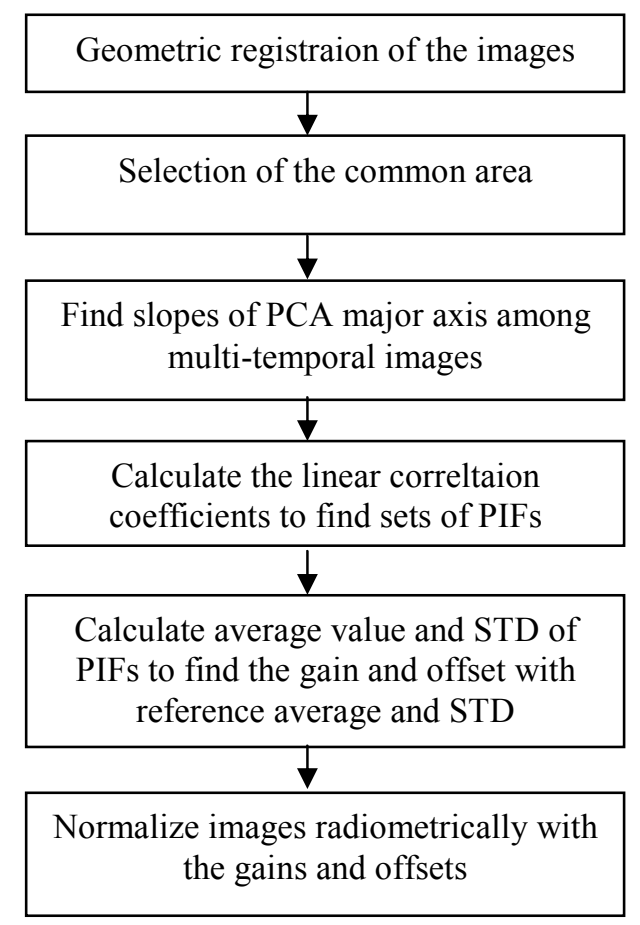

Fig.2. Flowchart of radiometric normalization procedure

\section{Case study and discusssion}

Automated haze removal section.A number of hazy images are tested with the Automated Adaptive Haze Removal System for experimentation. Although these images have different land covers (Urban and specific residential areas, agriculture, and mountain areas) and different regional occurrence of, again varying haze intensities, all haze was detected and removed applying the Automated Adaptive Haze Removal System. The operation is fully automatic, i.e. all parameters are not adjusted for each individual hazy image during processing (Figs.3 a, b, c and d). The results once more prove that the present algorithm of haze removal with topology based multiscale spatial analysis technology is valid and robust.
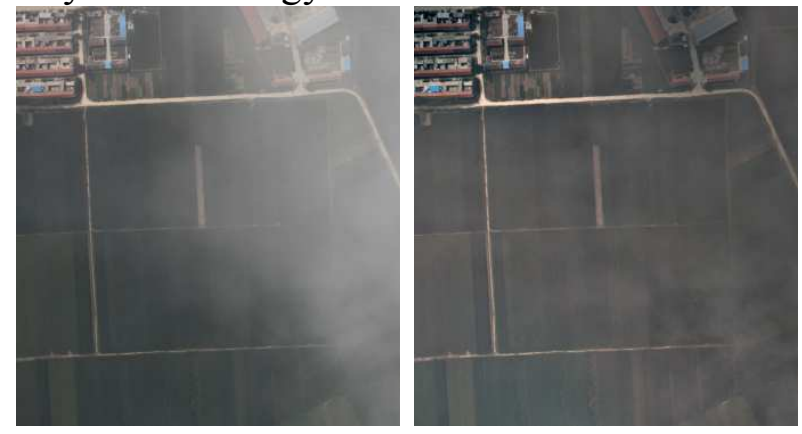

Fig. 3a. Fully automated haze removal in EO image

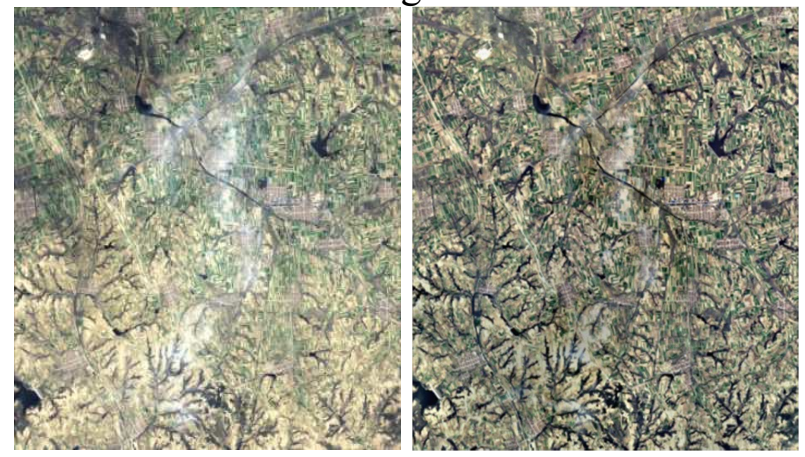

Fig. 3b. Fully automated haze removal in EO image
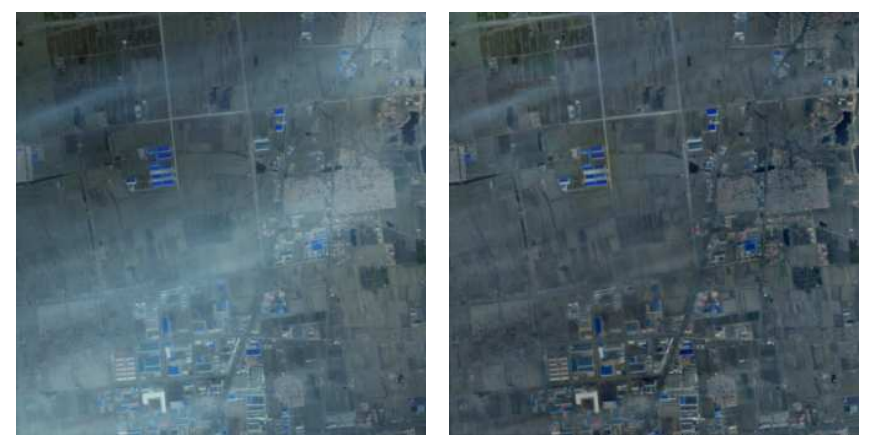

Fig. 3c. Fully automated haze removal in EO image
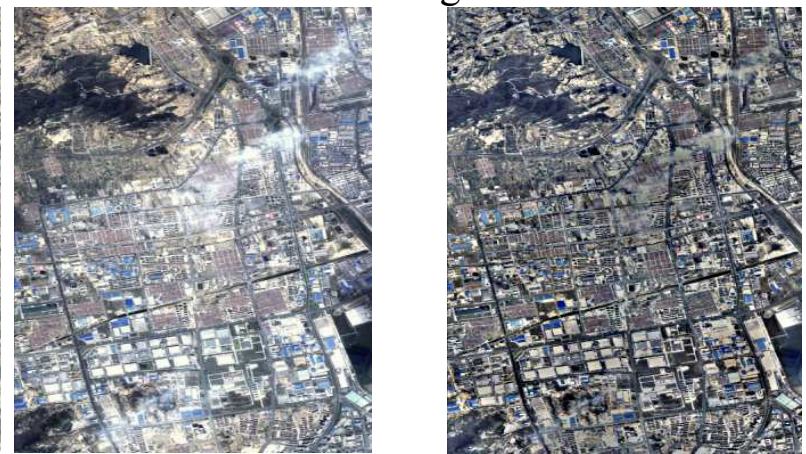

Fig. 3d. Fully automated haze removal in EO image 
The Adjustment section. This fully automatic procedure is mainly designed and developed to detect and remove the haze with typical spatial distribution, although it could also be used for thin cloud or fog removal with similar spatial distribution. Due to the complexity of atmospheric conditions, sometimes haze, cloud or fog may show mixing and create atypical spatial distribution patterns. For these cases, the adjustment section is designed for adjusting the parameters to fit the spatial features and specific haze intensity. This result proves that haze removal with adjustment is more effective(Figs.4 a, b and c).

It is proven from experiments that the procedure is suitable for quite a wide range of haze detection and removal, which includes different haze intensities, and different haze distribution on top of different land covers, urban or rural.

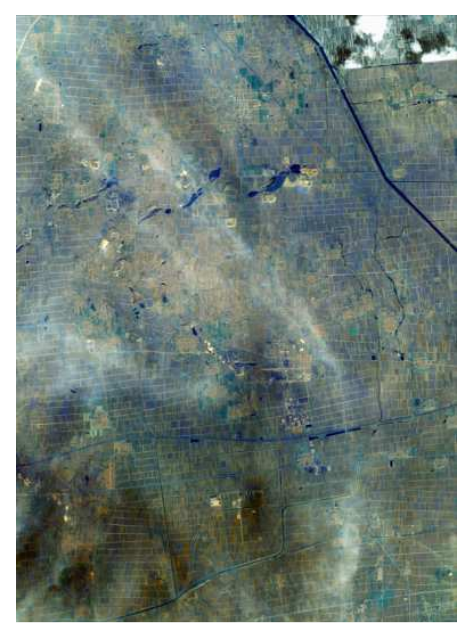

Fig. 4a. Original hazy image

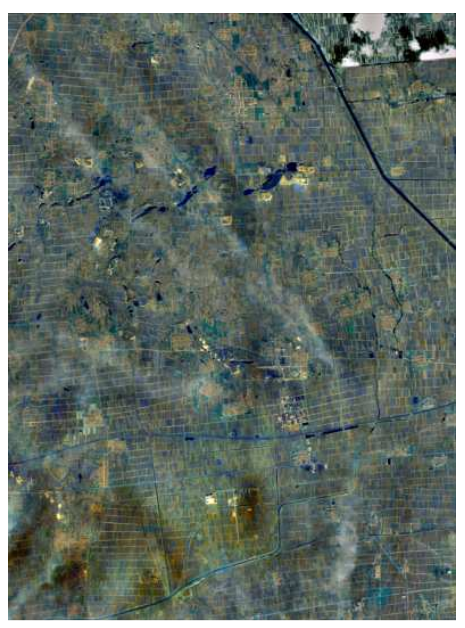

Fig. 4b. De-hazy image with automation method

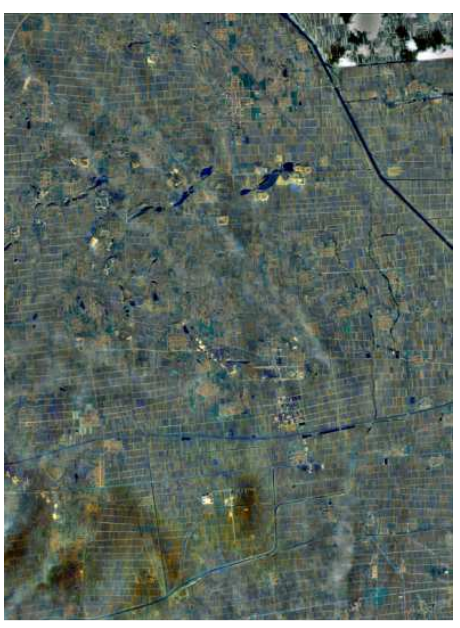

Fig. 4c. De-hazy image with adjustment method(haze feature:thinner,haze intensity:finer)

Radiometric normalization section. Three multitemporal QuickBird images are used from the same area (Figs.5 a,b and c).All images are first geometrically registered.The image2005 is convered by spatial varying haze. First, the haze of image 2005 was detected and removed applying the Automated Adaptive Haze Removal System.Then using radiometric normalization method, three images are normalized radiometrically to a common scale tied to the reference radiometric levels(Figs.6 a,b and c)。

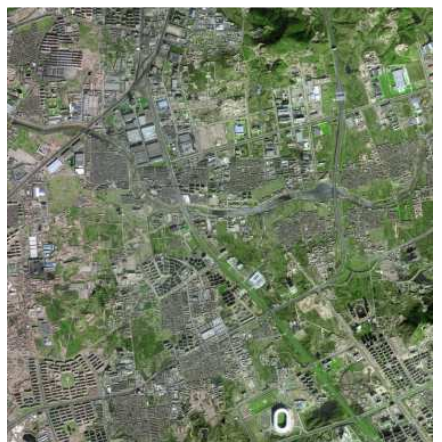

Fig.5a. Image2004

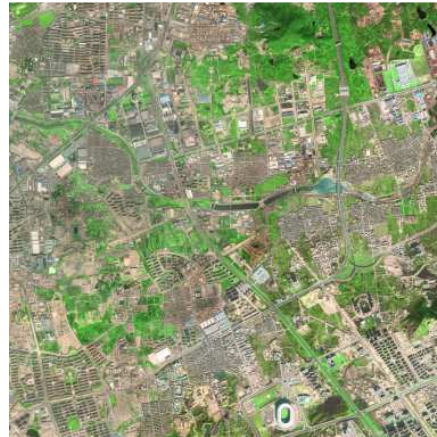

Fig.5b. Image2005

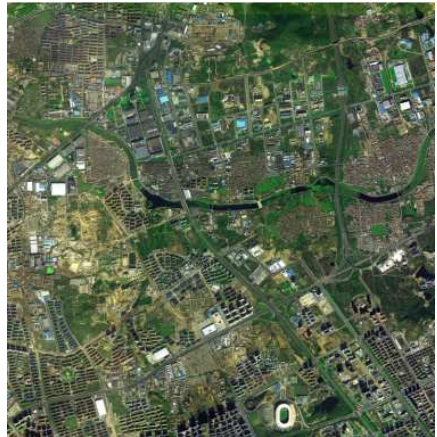

Fig.5c. Image2006 


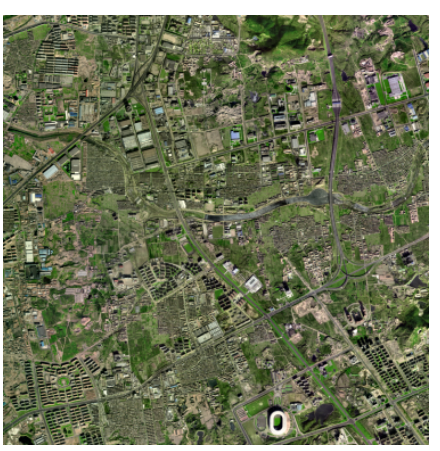

Fig.6a. Normalized image2004

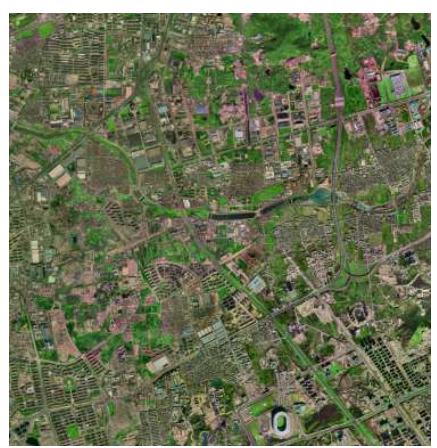

Fig.6b. Normalized and dehazy image 2005

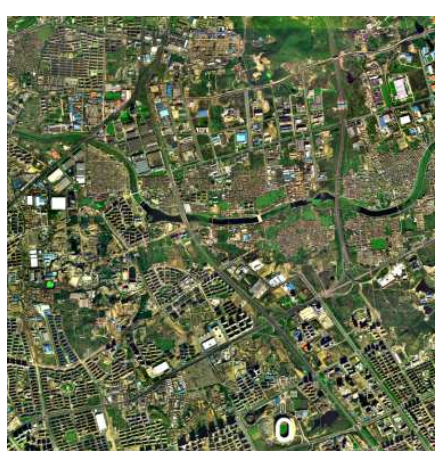

Fig.6c. Normalized image2006

\section{Summary}

The present study is focused on the applications of the innovative topology based multiscale spatial analysis technology, mainly haze removal in EO images. The experiments show the algorithms developed for haze removal are valid and robust. Meanwhile,the present procedure provides a simple and practical method for the radiometric normalization of multitemporal electro-optical images of the same area.

The high quality of haze removal in Electro Optical image will recover a considerable number of contaminated data resources. The applications of Electro Optical image data will be improved significantly with haze removal and radiometric normalization, especially for land cover change detection and quantitative analysis. The classification and segmentation in Electro Optical image will be improved as well.

Supported by International S\&T Cooperation, Project No.2010DFA71210

\section{References}

[1] D. Lu, Detection and substitution of clouds/hazes and their cast shadows on IKONOS images, Int.J. Remote Sensing, Vol.28,No.18(2007) 4027-4035.

[2] D. Tanre, C. Deroo, M. Duhaut, J. J. Morcrette, J. Perbos and P. Y. Deschamps, Description of a computer code to simulate the satellite signal in the solar spectrum: The $5 \mathrm{~S}$ code, Int. J. Remote Sensing, Vol.11, No. 2(1990)59-668.

[3] M. A. Spanner, L. L. Pierce, D. L. Peterson and S.W. Running, Remote sensing of temperate coniferous forest leaf area index: The influence of canopy closure, understory vegetation and background reflectance, Int. J. Remote Sensing, Vol. 11, No. 6(1990) 1469-1476.

[4] R. Richter, A fast atmospheric correction algorithm applied to Landsat TM images, Int. J. Remote Sensing, Vol. 11, No. 1(1990) 159-166.

[5] P. S. Chaez, Radiometric calibration of Landsat thematic and mapper multispectral images, Photorammetric Engineering and Remote Sensing, Vol.55, No.9(1989) 1285-1294.

[6] P. M. Teillet, G. Fedosejevs, On the dark target approach to atmospheric correction of remotely sensed data, Canadian Journal of Remote Sensing, Vol.21, No.4(1995) 374-387.

[7] G. Dal Moro and L. Halounova, Haze removal for high-resolution satellite data: A case study, International Journal of Remote Sensing, Vol.28,No.10(2007) 2187-2205.

[8] Y. Zhang, B.Guindon and J. Cihlar, An image transform to characterize and compensate for spatial variations in thin cloud contamination of Landsat images, Remote Sensing of Environment, Vol.82,No.2-3(2002) 173-187. 
[9] E.S. Artamonov,K.T.Protasov,Restoration of fragments of satellite images of the Rarth's underlying surface shadowed by haze and clouds, Proc. of SPIE Sixth International Symposium on Atmospheric and Ocean Optics, Vol.2983(1999) 170-178.

[10]R. Richter, A spatially adaptive fast atmospheric correction algorithm, Int. J. Remote Sensing, Vol.17(1996) 1201-1214.

[11]F. Chen, D. Yan and Z Zhao, Haze Detection and Removal in Remote Sensing Images Based on Undecimated Wavelet Transform, Geomatics and information science of Wuhan University, Vol.32,No.1(2007) 71-74.

[12]Y. Du, B. Guidon and J. Cihlar, Haze detection and removal in high resolution satellite image with wavelet analysis, IEEE Trans. Geoscoence and Remote Sensing, Vol.40, No.1(2002) 210217.

[13]J. Lavreau, De-haze landsat thematic mapper images, Photogramm. Eng. Remote Sensing, Vol. 57(1991) 1297-1302.

[14]Y. Du, M.Teillet and J.Cihlar, Radiometric normalizaion of multitemporal high resolution satellite images with quality control for land cover change detection, Remote Sensing of Environment,Vol.82(2002)123-134. 